quatrième série-tome 42 fascicule 2 mars-avril 2009

$$
\begin{aligned}
& \text { ANNALES } \\
& \text { SCIENTIFIQUES } \\
& \text { de } \\
& \text { L'ECOLE } \\
& \text { NORMALE } \\
& \text { SUPERIEURE }
\end{aligned}
$$

Pascal AUTISSIER

Géométrie, points entiers et courbes entières 


\title{
GÉOMÉTRIE, POINTS ENTIERS ET COURBES ENTIÈRES
}

\author{
PAR PASCAL AUTISSIER
}

\begin{abstract}
RÉSUMÉ. - Soit $X$ une variété projective sur un corps de nombres $K$ (resp. sur $\mathbb{C}$ ). Soit $H$ la somme de «suffisamment de diviseurs positifs » sur $X$. On montre que tout ensemble de points quasi-entiers (resp. toute courbe entière) dans $X-H$ est non Zariski-dense.
\end{abstract}

Abstract. - Let $X$ be a projective variety over a number field $K$ (resp. over $\mathbb{C}$ ). Let $H$ be the sum of "sufficiently many positive divisors" on $X$. We show that any set of quasi-integral points (resp. any integral curve) in $X-H$ is not Zariski dense.

\section{Introduction}

Soient $K$ un corps de nombres et $S$ un ensemble fini de places de $K$. On note $O_{K ; S}$ l'anneau des $S$-entiers de $K$. On s'intéresse dans cet article aux solutions dans $O_{K ; S}^{r}$ de systèmes d'équations du type

$$
\forall i \in\{1 ; \ldots ; n\} \quad F_{i}\left(x_{1} ; \ldots ; x_{r}\right)=0,
$$

où les $F_{i}$ sont des polynômes à $r$ variables et à coefficients dans $O_{K ; S}$. Pour formaliser cette étude, on utilise le langage de la géométrie algébrique :

Désignons par $Y$ la «variété algébrique » sur $K$ définie par $F_{1}=0, \ldots, F_{n}=0$. Tout ensemble de solutions de $(*)$ dans $O_{K ; S}^{r}$ définit alors un ensemble (de points) $S$-entier sur $Y$.

Le problème est de donner des conditions géométriques suffisantes sur $Y$ pour que tout ensemble $S$-entier soit non Zariski-dense dans $Y$.

Dans la suite, on se donne $Y$ sous la forme $Y=X-D$, où $X$ est une variété projective sur $K$ de dimension $d \geq 1$ et $D$ un diviseur effectif sur $X$. L'esprit de la conjecture de Lang et Vojta (cf. conjecture 4.2 de [9] p. 223) est qu'une telle condition suffisante s'exprime en termes de « positivité » de $D$ : 
Conjecture (Lang, Vojta). - Soit $X$ une variété projective lisse sur $K$ de diviseur canonique $\mathcal{K}_{X}$. Soit $D$ un diviseur effectif sur $X$, à croisements normaux. Posons $Y=X-D$. On suppose $\mathcal{K}_{X}+D$ gros (par exemple ample) sur $X$. Alors tout ensemble $S$-entier sur $Y$ est non Zariski-dense dans $Y$.

Les théorèmes de Siegel et de Faltings [5] montrent cette conjecture lorsque $X$ est une courbe. Plus généralement, cet énoncé est connu de Faltings [6] lorsque $X$ est une sous-variété de variété abélienne. Par ailleurs, c'est un corollaire direct du théorème du sous-espace lorsque $X=\mathbb{P}_{K}^{d}$ et $D$ égale la somme de $d+2$ hyperplans en position générale.

Notons cependant que la conjecture est encore largement ouverte : le cas où $X=\mathbb{P}_{K}^{2}$ n'est par exemple pas connu.

Dans cet article, on démontre des cas particuliers de cette conjecture, lorsque $D$ a «suffisamment » de composantes irréductibles. Plus précisément, disons qu'une variété $Y$ sur $K$ est arithmétiquement quasi-hyperbolique lorsqu'il existe un fermé $Z \neq Y$ tel que pour toute extension finie $K^{\prime}$ de $K$ et tout ensemble quasi-entier $\mathcal{E} \subset Y\left(K^{\prime}\right)$ sur $Y$, l'ensemble $\mathcal{E}-Z\left(K^{\prime}\right)$ soit fini (cf. section 2 pour les autres définitions). On prouve le résultat suivant :

ThÉorème 1.1. - Soit $X$ une variété projective sur $K$ de dimension $d \geq 2$. Soient $\delta$ un entier $\geq 2$ et $D_{1} ; \ldots ; D_{d \delta}$ des diviseurs effectifs presque amples sur $X$ qui se coupent proprement deux à deux. On suppose que toute intersection de $\delta+1$ quelconques d'entre eux est vide. Posons $Y=X-D_{1} \cup \cdots \cup D_{d \delta}$. Alors $Y$ est arithmétiquement quasi-hyperbolique. En particulier, tout ensemble $\mathcal{E} \subset Y(K) S$-entier sur $Y$ est non Zariski-dense dans $Y$.

Cet énoncé améliore un résultat récent de Levin (cf. théorème 10.4A de [11]). En fait, Levin a besoin de $2\left\lfloor\frac{\delta+1}{2}\right\rfloor d+1$ diviseurs au lieu de $d \delta$ (cf. aussi remarque 2.4 pour une comparaison des travaux).

On démontre en outre l'énoncé suivant (où $\lambda_{d}^{\prime}=\left[1-\left(1-\frac{1}{d}\right)^{d+1}\right]^{-1}\left(1+\frac{1}{d}\right)$ est une constante $\leq \frac{12}{7}$ ne dépendant que de $d$, cf. remarque 2.3$)$ :

ThÉorème 1.2. - Soit $X$ une variété projective sur $K$ de dimension $d \geq 2$. Soient $D_{1} ; \ldots ; D_{r}$ des diviseurs effectifs non nuls et nefs sur $X$ qui se coupent proprement (avec $\left.r>\lambda_{d}^{\prime} d\right)$. Posons $L=\sum_{i=1}^{r} D_{i}$ et $Y=X-D_{1} \cup \cdots \cup D_{r}$. On suppose que le $\mathbb{Q}$ diviseur $L-\lambda_{d}^{\prime} d D_{i}$ est ample pour tout $i \in\{1 ; \ldots ; r\}$. Alors $Y$ est arithmétiquement quasi-hyperbolique.

L'hypothèse sur les $L-\lambda_{d}^{\prime} d D_{i}$ est vérifiée lorsque les $D_{i}$ vivent dans un cône « suffisamment étroit » du groupe de Néron-Severi de $X$. L'intérêt de ce résultat réside dans le nombre (potentiellement linéaire en $d$ ) de diviseurs à considérer.

Appliquons, à titre d'exemple, le théorème 1.2 au cas où $X=\left(\mathbb{P}_{K}^{1}\right)^{d}(\operatorname{avec} d \geq 2)$ :

Soit $r$ un entier $>\lambda_{d}^{\prime} d$. Pour $i \in\{1 ; \ldots ; r\}$, soit $D_{i}$ un diviseur effectif non nul sur $\left(\mathbb{P}_{K}^{1}\right)^{d}$, de $d$-degré $\left(e_{i 1} ; \ldots ; e_{i d}\right)$.

Corollaire. - Supposons que les diviseurs $D_{1} ; \ldots ; D_{r}$ se coupent proprement et que l'on $a \lambda_{d}^{\prime} d \max _{i} e_{i j}<\sum_{i=1}^{r} e_{i j}$ pour tout $j \in\{1 ; \ldots ; d\}$. Alors $\left(\mathbb{P}_{K}^{1}\right)^{d}-D_{1} \cup \cdots \cup D_{r}$ est arithmétiquement quasi-hyperbolique. 
On observe qu'une application directe du corollaire 0.3 de Vojta [16] ne donne ce résultat que pour $r \geq 2 d+1$.

Remarquons que les théorèmes 1.1 et 1.2 s'inscrivent bien dans le cadre de la conjecture de Lang et Vojta, puisque si $X$ est lisse sur $K$ de diviseur canonique $\mathcal{K}_{X}$ et les $D_{i}$ sont amples $\operatorname{sur} X$, alors $\mathcal{K}_{X}+D_{1}+\cdots+D_{r}$ est ample sur $X$ dès que $r \geq d+2$ (c'est une conséquence du théorème du cône de Mori, cf. exemple 1.5.35 de [10] p. 87).

Les démonstrations reposent sur une extension (théorème 3.3) de travaux de CorvajaZannier [3] et de Levin [11], qui donne des conditions géométriques de non-Zariski-densité des points $S$-entiers, et sur un bon choix (théorème 4.4) de multiplicités associées aux diviseurs $D_{i}$.

L'ingrédient arithmétique principal est la version de Vojta [15] du théorème du sousespace de Schmidt [13] et Schlickewei [12] (c'est un énoncé d'approximation diophantienne qui généralise le théorème de Roth).

Par ailleurs, Vojta [14] a développé un « dictionnaire » entre la géométrie diophantienne et la théorie de Nevanlinna : l'étude des points $S$-entiers sur les variétés sur $K$ est mise en analogie avec l'étude des courbes entières sur les variétés complexes.

Pour étayer ce dictionnaire, on montre aussi les énoncés qui «correspondent » aux théorèmes 1.1 et 1.2 :

ThÉORÈme 1.3. - Soit $X$ une variété complexe projective de dimension $d \geq 2$. Soient $D_{1} ; \ldots ; D_{d \delta}$ des diviseurs effectifs presque amples sur $X$ qui se coupent proprement deux $\grave{a}$ deux (avec $\delta \geq 2$ ). On suppose que toute intersection de $\delta+1$ quelconques d'entre eux est vide. Posons $Y=X-D_{1} \cup \cdots \cup D_{d \delta}$. Alors $Y$ est Brody quasi-hyperbolique. En particulier, toute courbe entière $f: \mathbb{C} \rightarrow Y(\mathbb{C})$ est d'image non Zariski-dense dans $Y$.

ThÉORÈMe 1.4. - Soit $X$ une variété complexe projective de dimension $d \geq 2$. Soient $D_{1} ; \ldots ; D_{r}$ des diviseurs effectifs non nuls et nefs sur $X$ qui se coupent proprement (avec $\left.r>\lambda_{d}^{\prime} d\right)$. Posons $L=\sum_{i=1}^{r} D_{i}$ et $Y=X-D_{1} \cup \cdots \cup D_{r}$. On suppose que le $\mathbb{Q}$-diviseur $L-\lambda_{d}^{\prime} d D_{i}$ est ample pour tout $i \in\{1 ; \ldots ; r\}$. Alors $Y$ est Brody quasi-hyperbolique.

La section 2.1 décrit les résultats purement géométriques utilisés, qui sont prouvés aux sections 4 et 5 . La section 2.2 donne les critères de quasi-hyperbolicité, qui sont démontrés à la section 3 .

Je remercie Antoine Chambert-Loir et Christophe Mourougane pour de fructueuses discussions. Je remercie également le rapporteur pour ses suggestions pertinentes.

\section{Définitions et énoncés}

\subsection{Géométrie}

Soit $K$ un corps de caractéristique nulle. 
Conventions. - On appelle variété sur $K$ tout schéma quasi-projectif et géométriquement intègre sur $K$. Le mot « diviseur » sous-entend « diviseur de Cartier».

Soit $X$ une variété projective sur $K$ de dimension $d \geq 1$. Lorsque $L$ est un diviseur sur $X$ tel que $h^{0}(X ; L) \geq 1$, on désigne par $\mathbf{B}_{L}$ le lieu de base de $\Gamma(X ; L)$ et par $\Phi_{L}: X-\mathbf{B}_{L} \rightarrow$ $\mathbb{P}(\Gamma(X ; L))$ le morphisme défini par $\Gamma(X ; L)$. Pour tout diviseur effectif $D$ sur $X$, on note $1_{D}$ la section globale de $\mathcal{O}_{X}(D)$ qu'il définit.

DÉfinition. - Un diviseur $L$ sur $X$ est dit libre lorsque $\mathbf{B}_{L}$ est vide.

DÉfinition. - Un diviseur $L$ sur $X$ est dit gros lorsque $\liminf _{n \rightarrow+\infty} \frac{1}{n^{d}} h^{0}(X ; n L)>0$.

Définition. - Soit $L$ un diviseur gros sur $X$. On dit que $L$ est presque ample lorsqu'il existe un entier $n \geq 1$ tel que $n L$ soit libre.

DÉfinition. - Un $\mathbb{R}$-diviseur $L$ sur $X$ est dit nef lorsque pour tout 1-cycle effectif $C$ sur $X$, on a $\langle L . C\rangle \geq 0$ (où $\langle L . C\rangle$ désigne le nombre d'intersection).

Définition. - Soient $D_{1}$ et $D_{2}$ deux diviseurs effectifs sur $X$. On dit que $D_{1}$ et $D_{2}$ se coupent proprement lorsque $\mathcal{O}_{X}\left(-D_{1}-D_{2}\right)=\mathcal{O}_{X}\left(-D_{1}\right) \cap \mathcal{O}_{X}\left(-D_{2}\right)$.

Définition. - Plus généralement, soient $D_{1} ; \ldots ; D_{r}$ des diviseurs effectifs sur $X$. On dit que $D_{1} ; \ldots ; D_{r}$ se coupent proprement lorsque, pour toute partie $I$ non vide de $\{1 ; \ldots ; r\}$, la section globale $\left(1_{D_{i}}\right)_{i \in I}$ de $\bigoplus_{i \in I} \mathcal{O}_{X}\left(D_{i}\right)$ est régulière (autrement dit, pour tout $x \in \bigcap_{i \in I} D_{i}$, en notant $\varphi_{i}$ une équation locale de $D_{i}$ en $x$, les $\left(\varphi_{i}\right)_{i \in I}$ forment une suite régulière de l'anneau local $\mathcal{O}_{X ; x}$ ).

Remarque. - Supposons $X$ de Cohen-Macaulay (par exemple lisse sur $K$ ); alors d'après le lemme A.7.1 de [7] p. 418, les diviseurs $D_{1} ; \ldots ; D_{r}$ se coupent proprement si et seulement si, pour toute partie $I$ non vide de $\{1 ; \ldots ; r\}$, le fermé $\bigcap_{i \in I} D_{i}$ est purement de codimension \# $I$ dans $X$ (éventuellement vide).

Soit $L$ un diviseur sur $X$ tel que $h^{0}(X ; L) \geq 1$. Soient $D_{1} ; \ldots ; D_{r}$ des diviseurs effectifs non nuls $\operatorname{sur} X$ (avec $r \geq 1$ ). Notons $\mathcal{P}$ l'ensemble des parties $I$ non vides de $\{1 ; \ldots ; r\}$ telles que $\bigcap_{i \in I} D_{i}$ soit non vide. Pour $I \in \mathcal{P}, \underline{a}=\left(a_{i}\right)_{i} \in \mathbb{N}^{I}$ et $k \in \mathbb{N}^{*}$, on définit le sous-espace vectoriel $V_{I ; a ; k}$ de $\Gamma(X ; L)$ par

$$
V_{I ; \underline{a} ; k}=\sum_{\underline{b}} \Gamma\left(X ; L-\sum_{i \in I} b_{i} D_{i}\right) \text { où la somme porte sur les } \underline{b} \in \mathbb{N}^{I} \text { tels que } \sum_{i \in I} a_{i} b_{i} \geq k .
$$

DÉfinition. - On pose

$$
\nu\left(L ; D_{1} ; \ldots ; D_{r}\right)=\inf _{I \in \mathcal{P}} \inf _{\underline{a} \in \mathbb{N}^{I}-\{0\}} \frac{\sum_{k \geq 1} \operatorname{dim} V_{I ; \underline{a} ; k}}{h^{0}(X ; L) \sum_{i \in I} a_{i}} .
$$

On démontre à la section 4 le résultat suivant :

ThÉorème 2.1. - On suppose $d \geq 2$. Soient $D_{1} ; \ldots ; D_{r}$ des diviseurs effectifs presque amples sur $X$ qui se coupent proprement deux à deux; supposons que toute intersection de $\delta+1$ quelconques d'entre eux est vide (avec $2 \leq \delta \leq r$ ). Il existe alors $\left(m_{1} ; \ldots ; m_{r}\right) \in \mathbb{N}^{* r}$ tel qu'en posant $L=\sum_{i=1}^{r} m_{i} D_{i}$, on ait $\liminf _{n \rightarrow+\infty} \frac{1}{n} \nu\left(n L ; m_{1} D_{1} ; \ldots ; m_{r} D_{r}\right)>\frac{r}{d \delta}$. 
Posons $\lambda_{d}=\left[1-\left(1-\frac{1}{d}\right)^{d+1}\right] \frac{d}{d+1}$ et $\lambda_{d}^{\prime}=\frac{1}{\lambda_{d}}$. On prouve à la section 5.2 l'énoncé suivant :

ThÉORÈme 2.2. - Soient $D_{1} ; \ldots ; D_{r}$ des diviseurs effectifs non nuls et nefs sur $X$ qui se coupent proprement. On suppose que $L=\sum_{i=1}^{r} D_{i}$ est ample. Soit $\theta>1$ un réel tel que le $\mathbb{R}$-diviseur $L-d \theta D_{i}$ soit nef pour tout $i \in\{1 ; \ldots ; r\}$. On a alors la minoration

$$
\liminf _{n \rightarrow+\infty} \frac{1}{n} \nu\left(n L ; D_{1} ; \ldots ; D_{r}\right) \geq \lambda_{d} \theta .
$$

Remarque 2.3. - La suite $\left(\lambda_{d}^{\prime}\right)_{d \geq 2}$ est décroissante (on le voit en écrivant la relation $\left.\lambda_{d}=\int_{0}^{1}\left(1-\frac{t}{d}\right)^{d} \mathrm{~d} t\right)$. En outre, $\lambda_{d}$ converge vers $1-e^{-1}$ lorsque $d$ tend vers $+\infty$.

\subsection{Hyperbolicité}

Lorsque $K$ est un corps de nombres et $S$ un ensemble fini de places de $K$, on note $O_{K ; S}$ l'anneau des $S$-entiers de $K$, i.e. l'ensemble des $x \in K$ tels que $|x|_{v} \leq 1$ pour toute place finie $v \notin S$.

Soit $K$ un corps de nombres.

DÉfinition. - Soient $Y$ une variété sur $K, K^{\prime}$ une extension finie de $K$ et $S$ un ensemble fini de places de $K^{\prime}$. Un ensemble $\mathcal{E} \subset Y\left(K^{\prime}\right)$ est dit $S$-entier sur $Y$ lorsqu'il existe un $O_{K^{\prime} ; S^{-}}$ schéma intègre et quasi-projectif $\mathcal{Y}$ de fibre générique $Y_{K^{\prime}}$ tel que $\mathcal{E} \subset \mathcal{Y}\left(O_{K^{\prime} ; S}\right)$.

DÉfinition. - Soient $Y$ une variété sur $K$ et $K^{\prime}$ une extension finie de $K$. Un ensemble $\mathcal{E} \subset Y\left(K^{\prime}\right)$ est dit quasi-entier sur $Y$ lorsqu'il existe un ensemble fini $S$ de places de $K^{\prime}$ tel que $\mathcal{E}$ soit $S$-entier sur $Y$.

Définition. - Soit $Y$ une variété sur $K$. On dit que $Y$ est arithmétiquement quasihyperbolique lorsqu'il existe un fermé $Z \neq Y$ tel que, pour toute extension finie $K^{\prime}$ de $K$ et tout ensemble quasi-entier $\mathcal{E} \subset Y\left(K^{\prime}\right)$ sur $Y$, l'ensemble $\mathcal{E}-Z\left(K^{\prime}\right)$ soit fini.

DÉfinition. - Soit $Y$ une variété complexe. Une courbe entière sur $Y$ est une application holomorphe $f: \mathbb{C} \rightarrow Y(\mathbb{C})$ non constante.

Définition. - Soit $Y$ une variété complexe. On dit que $Y$ est Brody quasi-hyperbolique lorsqu'il existe un fermé $Z \neq Y$ tel que pour toute courbe entière $f$ sur $Y$, on ait $f(\mathbb{C}) \subset$ $Z(\mathbb{C})$.

L'intérêt de la définition de $\nu$ réside dans les critères suivants :

ThÉorème (3.3). - Soit $X$ une variété projective sur $K$ de dimension $d \geq 1$. Soient $D_{1} ; \ldots ; D_{r}$ des diviseurs effectifs non nuls sur $X$ qui se coupent proprement deux à deux. Posons $Y=X-D_{1} \cup \cdots \cup D_{r}$. Soit $n \geq 1$ un entier. On suppose que le diviseur $L=n \sum_{i=1}^{r} D_{i}$ est libre et gros sur $X$ et que $\nu\left(L ; D_{1} ; \ldots ; D_{r}\right)>n$. Alors $Y$ est arithmétiquement quasihyperbolique. 
Théorème (3.5). - Soit $X$ une variété complexe projective de dimension $d \geq 1$. Soient $D_{1} ; \ldots ; D_{r}$ des diviseurs effectifs non nuls sur $X$ qui se coupent proprement deux à deux. Posons $Y=X-D_{1} \cup \cdots \cup D_{r}$. Soit $n \geq 1$ un entier. On suppose que le diviseur $L=n \sum_{i=1}^{r} D_{i}$ est libre et gros sur $X$ et que $\nu\left(L ; D_{1} ; \ldots ; D_{r}\right)>n$. Alors $Y$ est Brody quasi-hyperbolique.

On en déduit le théorème 1.1 - respectivement 1.3 - en appliquant le théorème 2.1 (avec $r=d \delta$ ) puis le théorème 3.3 - respectivement 3.5 - .

On en déduit de même les théorèmes 1.2 et 1.4 en appliquant le théorème 2.2.

Remarque 2.4. - Notons ici $\mathcal{L}$ l'ensemble des bases de $\Gamma(X ; L), \mathcal{P}$ l'ensemble des parties $I$ non vides de $\{1 ; \ldots ; r\}$ telles que $\bigcap_{i \in I} D_{i}$ soit non vide, et posons

$$
\nu^{\prime}\left(L ; D_{1} ; \ldots ; D_{r}\right)=\frac{1}{h^{0}(X ; L)} \inf _{I \in \mathcal{P}} \sup _{\mathcal{B} \in \mathcal{L}} \inf _{i \in I} \sum_{s \in \mathcal{B}} \mu_{i}(s),
$$

où $\mu_{i}(s)$ désigne le plus grand entier $\mu$ tel que le diviseur $\operatorname{div}(s)-\mu D_{i}$ soit effectif (i.e. «l'ordre d'annulation de $s$ en $\left.D_{i} »\right)$. Levin (cf. section 8 de [11]) donne ces résultats de quasihyperbolicité lorsque $X$ est lisse et $\nu^{\prime}\left(L ; D_{1} ; \ldots ; D_{r}\right)>n$. Les énoncés ci-dessus sont plus généraux, puisqu'un peu d'algèbre linéaire montre que $\nu\left(L ; D_{1} ; \ldots ; D_{r}\right) \geq \nu^{\prime}\left(L ; D_{1} ; \ldots ; D_{r}\right)$ (et $X$ n'est pas supposée lisse).

\section{Démonstration des critères}

\subsection{Rappels}

Soit $X$ une variété complexe projective. Soit $L$ un faisceau inversible sur $X$. On munit $L$ d'une métrique (continue) \|\| et on pose $\hat{L}=(L ;\|\|)$.

Soit $f$ une courbe entière sur $X$. On définit la fonction caractéristique $\mathrm{T}_{\hat{L} ; f}: \mathbb{R}_{+} \rightarrow \mathbb{R}$ de $f$ relativement à $\hat{L}$ de la manière suivante :

On choisit une section rationnelle $s$ de $L$ définie et non nulle en $f(0)$. Pour tout réel $r \geq 0$, on pose

$$
\mathrm{T}_{\hat{L} ; f}(r)=\sum_{|z| \leq r} \mu_{z}\left(f^{*} s\right) \ln \frac{r}{|z|}-\int_{0}^{2 \pi} \ln \left\|s\left(f\left(r e^{i \theta}\right)\right)\right\| \frac{\mathrm{d} \theta}{2 \pi}+\ln \|s(f(0))\|,
$$

où $\mu_{z}\left(f^{*} s\right)$ désigne l'ordre de $f^{*} s$ en $z \in \mathbb{C}$. Cela ne dépend pas du choix de $s$.

Soient $K$ un corps de nombres et $X^{\prime}$ une variété projective sur $K$. Soit $L^{\prime}$ un faisceau inversible sur $X^{\prime}$. On munit $L^{\prime}$ d'une métrique adélique $\left(\|\|_{v}\right)_{v}$ et on pose $\hat{L}^{\prime}=\left(L^{\prime} ;\left(\|\|_{v}\right)_{v}\right)$ (pour des précisions sur les métriques adéliques, on pourra consulter le paragraphe $1.2 \mathrm{de}$ [18]).

Soient $K^{\prime}$ une extension finie de $K$ et $P \in X^{\prime}\left(K^{\prime}\right)$. On définit la hauteur (normalisée) $\mathrm{h}_{\hat{L}^{\prime}}(P)$ de $P$ relativement à $\hat{L}^{\prime}$ de la façon suivante :

On choisit une section rationnelle $s^{\prime}$ de $L^{\prime}$ définie et non nulle en $P$. On pose

$$
\mathrm{h}_{\hat{L}^{\prime}}(P)=-\frac{1}{\left[K^{\prime}: \mathbb{Q}\right]} \sum_{v} \ln \left\|s^{\prime}(P)\right\|_{v}
$$

où $v$ parcourt l'ensemble des places de $K^{\prime}$. Ce réel ne dépend pas du choix de $s^{\prime}$.

$4^{\mathrm{e}}$ SÉRIE - TOME $42-2009-\mathrm{N}^{\mathrm{o}} 2$ 


\subsection{Cas arithmétique}

Commençons par un résultat facile d'algèbre linéaire :

Lemme 3.1. - Soient $K$ un corps et $V$ un $K$-espace vectoriel de dimension finie. Soit $\left(\mathcal{F}_{k}\right)_{k \geq 1}$ une suite décroissante de parties de $V$ telle que $\mathcal{F}_{k}=\{0\}$ pour tout $k$ assez grand. Il existe alors une base $\mathcal{B}$ de $V$ adaptée à la suite $\left(\mathcal{F}_{k}\right)_{k \geq 1}$, i.e. $\mathcal{B} \cap \mathcal{F}_{k}$ est une base $\operatorname{de} \operatorname{Vect}\left(\mathcal{F}_{k}\right)$ pour tout $k \geq 1$.

Démonstration. - Soit $m \geq 1$ un entier tel que $\mathcal{F}_{k}=\{0\}$. On pose $\mathcal{B}_{m}=\varnothing$. On construit par récurrence une suite $\left(\mathcal{B}_{m} ; \ldots ; \mathcal{B}_{1}\right)$ de parties de $V$ de la manière suivante :

Pour $k \in\{1 ; \ldots ; m-1\}$, on complète la partie libre $\mathcal{B}_{k+1}$ en une base $\mathcal{B}_{k} \operatorname{de} \operatorname{Vect}\left(\mathcal{F}_{k}\right)$ contenue dans $\mathcal{F}_{k}$.

Pour finir, on complète $\mathcal{B}_{1}$ en une base $\mathcal{B}$ de $V$.

Soient $K$ un corps de nombres et $X$ une variété projective sur $K$ de dimension $d \geq 1$. On va utiliser la version suivante du théorème du sous-espace de Schmidt, Schlickewei et Vojta :

Proposition 3.2. - Soit $L \in \operatorname{Pic}(X)$ libre et gros. Notons $q=h^{0}(X ; L)$. On munit $L$ d'une métrique adélique $\left(\|\|_{v}\right)_{v}$. Soient $s_{1} ; \ldots ; s_{N}$ des sections non nulles engendrant $\Gamma(X ; L)$. Soit $\varepsilon>0$. Il existe alors un fermé $Z \neq X$ tel que pour toute extension finie $K^{\prime}$ de $K$ et tout ensemble fini $S$ de places de $K^{\prime}$, l'ensemble des points $P \in(X-Z)\left(K^{\prime}\right)$ vérifiant

$$
\sum_{v \in S} \max _{J \in \mathcal{L}} \sum_{j \in J} \ln \left\|s_{j}(P)\right\|_{v}^{-1} \geq(q+q \varepsilon)\left[K^{\prime}: \mathbb{Q}\right] \mathrm{h}_{\hat{L}}(P)
$$

est fini, où $\mathcal{L}$ désigne l'ensemble des parties $J$ de $\{1 ; \ldots ; N\}$ telles que $\left(s_{j}\right)_{j \in J}$ soit une base de $\Gamma(X ; L)$.

Démonstration. - En posant $V=\Gamma(X ; L)$, on a un morphisme $\Phi_{L}: X \rightarrow \mathbb{P}(V)$ génériquement fini. Il existe donc un fermé $Z_{1} \neq X$ tel que $\Phi_{L \mid X-Z_{1}}$ soit à fibres finies. On applique alors la version de Vojta (cf. théorème 0.3 et reformulation 3.4 de [15]) du théorème du sous-espace :

Il existe une réunion finie $H$ de $K$-hyperplans de $\mathbb{P}(V) \simeq \mathbb{P}_{K}^{q-1}$ telle que pour toute extension finie $K^{\prime}$ de $K$ et tout ensemble fini $S$ de places de $K^{\prime}$, l'ensemble des points $P \in$ $\left(X-Z_{1} \cup \Phi_{L}^{-1}(H)\right)\left(K^{\prime}\right)$ vérifiant (1) est fini.

RemARQUe. - Vojta a en fait montré que l'on peut trouver un $Z$ indépendant de $\varepsilon$, mais on n'en aura pas besoin dans la suite.

On montre ci-dessous une extension d'un résultat de Levin (cf. théorème 8.3A de [11]), luimême inspiré de travaux de Corvaja et Zannier (cf. théorème principal de [3] p. 707-708) :

ThÉORÈme 3.3. - Soient $D_{1} ; \ldots ; D_{r}$ des diviseurs effectifs non nuls sur $X$ qui se coupent proprement deux à deux. Posons $Y=X-D_{1} \cup \cdots \cup D_{r}$. Soit $m \geq 1$ un entier. On suppose que le diviseur $L=m \sum_{i=1}^{r} D_{i}$ est libre et gros sur $X$ et que $\nu\left(L ; D_{1} ; \ldots ; D_{r}\right)>m$. Alors $Y$ est arithmétiquement quasi-hyperbolique.

Démonstration. - On procède en deux étapes : dans la première, on construit un fermé $Z \neq X$ candidat à contenir «presque tous les points entiers »; dans la seconde, on prouve que $Y$ est arithmétiquement quasi-hyperbolique. 
Étape 1. - On pose $\varepsilon=\frac{1}{4 m}\left(\nu\left(L ; D_{1} ; \ldots ; D_{r}\right)-m\right)$ et $q=h^{0}(X ; L)$, on choisit un entier $c \geq 1$ tel que $h^{0}\left(X ; L-c D_{i}\right)=0$ pour tout $i \in\{1 ; \ldots ; r\}$, et on fixe un entier $b \geq \frac{c r}{m \varepsilon}$. Choisissons aussi une base $\mathcal{B}_{0}$ de $\Gamma(X ; L)$.

Désignons par $\mathcal{P}$ l'ensemble des parties $I$ non vides de $\{1 ; \ldots ; r\}$ telles que $\bigcap_{i \in I} D_{i}$ soit non vide. Soit $I \in \mathcal{P}$. On note $\Delta_{I}$ l'ensemble des $\underline{a}=\left(a_{i}\right)_{i} \in \mathbb{N}^{I}$ tels que $\sum_{i \in I} a_{i}=b$. Soit $\underline{a} \in \Delta_{I}$. Pour $k \in \mathbb{N}^{*}$, on pose $\mathcal{F}_{k}=\bigcup_{\underline{b}} \Gamma\left(X ; L-\sum_{i \in I} b_{i} D_{i}\right)$ où la réunion porte sur les $\underline{b} \in \mathbb{N}^{I}$ tels que $\sum_{i \in I} a_{i} b_{i} \geq k$, et on note $V_{I ; \underline{a} ; k}=\operatorname{Vect}\left(\mathcal{F}_{k}\right)$. Le lemme 3.1 fournit une base $\mathcal{B}_{I ; \underline{a}}$ de $\Gamma(X ; L)$ adaptée à la suite $\left(\mathcal{F}_{k}\right)_{k \geq 1}$.

On munit chaque faisceau $\mathcal{O}_{X}\left(D_{i}\right)$ d'une métrique adélique. Appliquons le théorème du sous-espace (proposition 3.2) avec $\left\{s_{1} ; \ldots ; s_{N}\right\}=\mathcal{B}_{0} \cup \bigcup_{I ; \underline{a}} \mathcal{B}_{I ; \underline{a}}$ (remarquons que cette réunion est finie puisque $\mathcal{P}$ et les $\Delta_{I}$ le sont) :

Il existe un fermé $Z \neq X$ tel que pour toute extension finie $K^{\prime}$ de $K$ et tout ensemble fini $S$ de places de $K^{\prime}$, l'ensemble des points $P \in(X-Z)\left(K^{\prime}\right)$ vérifiant l'inégalité (1) est fini.

Étape 2. - Soient $K^{\prime}$ une extension finie de $K$ et $S$ un ensemble fini de places de $K^{\prime}$ contenant les places archimédiennes. Soit $\mathcal{E} \subset Y\left(K^{\prime}\right)$ un ensemble $S$-entier sur $Y$. Raisonnons par l'absurde en supposant $\mathcal{E}-Z\left(K^{\prime}\right)$ infini. On choisit une suite injective $\left(P_{n}\right)_{n \geq 0}$ d'éléments de $\mathcal{E}-Z\left(K^{\prime}\right)$.

Quitte à extraire, on peut supposer (par compacité) que pour tout $v \in S$, la suite $\left(P_{n v}\right)_{n \geq 0}$ converge dans $X\left(K_{v}^{\prime}\right)$ vers un $y_{v} \in X\left(K_{v}^{\prime}\right)$.

Pour tout $v \in S$, on note $I_{v}$ l'ensemble des $i \in\{1 ; \ldots ; r\}$ tels que $y_{v} \in D_{i}$. Quitte à extraire de nouveau, on peut supposer que pour tout $v \in S$ tel que $I_{v}$ soit non vide et tout $i \in I_{v}$, la suite $\left(\frac{\ln \left\|1_{D_{i}}\left(P_{n}\right)\right\|_{v}}{\sum_{j \in I_{v}} \ln \left\|1_{D_{j}}\left(P_{n}\right)\right\|_{v}}\right)_{n \geq 0}$ converge vers un $t_{v i} \in[0 ; 1]$. Remarquons que l'on a $\sum_{i \in I_{v}} t_{v i}=1$.

FaIt. - Soit $v \in S$. Il existe une base $\left(s_{1 v} ; \ldots ; s_{q v}\right)$ de $\Gamma(X ; L)$ contenue dans $\left\{s_{1} ; \ldots ; s_{N}\right\}$ telle que l'on ait la minoration suivante pour tout $n \geq 0$ :

$$
-\sum_{k=1}^{q} \ln \left\|s_{k v}\left(P_{n}\right)\right\|_{v} \geq-(q+2 q \varepsilon) \ln \left\|1_{L}\left(P_{n}\right)\right\|_{v}-O(1),
$$

où le $O(1)$ est indépendant de $n$.

Prouvons ce fait. Si $I_{v}$ est vide, on prend $\left\{s_{1 v} ; \ldots ; s_{q v}\right\}=\mathcal{B}_{0}$ et on obtient la minoration (2) en remarquant que $\ln \left\|1_{L}\left(P_{n}\right)\right\|_{v}=O(1)$ (puisque $y_{v} \notin L$ ).

On suppose maintenant $I_{v}$ non vide. On a donc $I_{v} \in \mathcal{P}$. Choisissons un $\underline{a}_{v}=\left(a_{v i}\right)_{i} \in \Delta_{I_{v}}$ tel que $\left|b t_{v i}-a_{v i}\right| \leq 1$ pour tout $i \in I_{v}$. On prend alors $\left\{s_{1 v} ; \ldots ; s_{q v}\right\}=\mathcal{B}_{I_{v} ; \underline{a}_{v}}$. Vérifions que ce choix convient.

Soit $s \in \Gamma(X ; L)-\{0\}$. Pour tout $i \in\{1 ; \ldots ; r\}$, notons $\mu_{i}(s)$ le plus grand entier $\mu$ tel que le diviseur $\operatorname{div}(s)-\mu D_{i}$ soit effectif. Puisque les diviseurs $D_{i}$ se coupent proprement deux à deux, le diviseur $\operatorname{div}(s)-\sum_{i \in I_{v}} \mu_{i}(s) D_{i}$ est encore effectif. Ceci implique

$$
-\ln \left\|s\left(P_{n}\right)\right\|_{v} \geq-\sum_{i \in I_{v}} \mu_{i}(s) \ln \left\|1_{D_{i}}\left(P_{n}\right)\right\|_{v}-O(1) .
$$


En remarquant que $t_{v i}>\frac{a_{v i}}{b}-\frac{2 m \varepsilon}{c r}$ et que $\mu_{i}(s) \leq c$ pour tout $i \in I_{v}$, on a, par définition des $t_{v i}$ :

$$
-\mu_{i}(s) \ln \left\|1_{D_{i}}\left(P_{n}\right)\right\|_{v} \geq-\left(\frac{a_{v i}}{b} \mu_{i}(s)-\frac{2 m \varepsilon}{r}\right) \sum_{j \in I_{v}} \ln \left\|1_{D_{j}}\left(P_{n}\right)\right\|_{v}
$$

pour tout $n$ assez grand et tout $i \in I_{v}$.

On en déduit l'inégalité (pour tout $n \geq 0$ )

$$
-\ln \left\|s\left(P_{n}\right)\right\|_{v} \geq-\left(\frac{1}{b} \sum_{i \in I_{v}} a_{v i} \mu_{i}(s)-2 m \varepsilon\right) \sum_{j \in I_{v}} \ln \left\|1_{D_{j}}\left(P_{n}\right)\right\|_{v}-O(1) .
$$

On écrit cette inégalité pour $s=s_{k v}$, puis on somme sur $k$. En observant que, pour $i \in I_{v}$, on a

$$
\begin{aligned}
\sum_{k=1}^{q} \sum_{i \in I_{v}} a_{v i} \mu_{i}\left(s_{k v}\right) & =\sum_{\mu \geq 1} \#\left\{k \in\{1 ; \ldots ; q\} \mid \sum_{i \in I_{v}} a_{v i} \mu_{i}\left(s_{k v}\right) \geq \mu\right\} \\
& =\sum_{\mu \geq 1} \operatorname{dim} V_{I_{v} ; \underline{a}_{v} ; \mu} \geq \nu\left(L ; D_{1} ; \ldots ; D_{r}\right) q b=(1+4 \varepsilon) q b m
\end{aligned}
$$

on trouve alors

$$
-\sum_{k=1}^{q} \ln \left\|s_{k v}\left(P_{n}\right)\right\|_{v} \geq-(q+2 q \varepsilon) m \sum_{j \in I_{v}} \ln \left\|1_{D_{j}}\left(P_{n}\right)\right\|_{v}-O(1) .
$$

Le fait énoncé (2) s'en déduit en remarquant que $\ln \left\|1_{D_{j}}\left(P_{n}\right)\right\|_{v}=O(1)$ pour tout $j \notin I_{v}$.

Maintenant, l'ensemble $\mathcal{E}$ est $S$-entier sur $Y$, donc pour tout $n \geq 0$, on a

$$
\left[K^{\prime}: \mathbb{Q}\right] \mathrm{h}_{\hat{L}}\left(P_{n}\right)=-\sum_{v \in S} \ln \left\|1_{L}\left(P_{n}\right)\right\|_{v}+O(1) .
$$

En utilisant la minoration (2), on obtient (pour tout $n \geq 0$ )

$$
-\sum_{v \in S} \sum_{k=1}^{q} \ln \left\|s_{k v}\left(P_{n}\right)\right\|_{v} \geq(q+2 q \varepsilon)\left[K^{\prime}: \mathbb{Q}\right] \mathrm{h}_{\hat{L}}\left(P_{n}\right)-O(1) .
$$

D'où une contradiction avec (1).

\subsection{Cas analytique}

Soit $X$ une variété complexe projective de dimension $d \geq 1$.

Proposition 3.4. - Soit $L \in \operatorname{Pic}(X)$ libre et gros. Notons $q=h^{0}(X ; L)$. On munit $L$ d'une métrique \|\| . Soient $s_{1} ; \ldots ; s_{N}$ des sections non nulles engendrant $\Gamma(X ; L)$. Soit $\varepsilon>0$. Il existe alors un fermé $Z \neq X$ tel que pour toute courbe entière $f$ sur $X$ d'image non contenue dans $Z(\mathbb{C})$, l'ensemble des réels $r \geq 0$ vérifiant

$$
\int_{0}^{2 \pi} \max _{J \in \mathcal{L}} \sum_{j \in J} \ln \left\|s_{j}\left(f\left(r e^{i \theta}\right)\right)\right\|^{-1} \frac{\mathrm{d} \theta}{2 \pi} \geq(q+q \varepsilon) \mathrm{T}_{\hat{L} ; f}(r)
$$

est de mesure de Lebesgue finie, où $\mathcal{L}$ désigne l'ensemble des parties $J$ de $\{1 ; \ldots ; N\}$ telles que $\left(s_{j}\right)_{j \in J}$ soit une base de $\Gamma(X ; L)$. 
Démonstration. - En posant $V=\Gamma(X ; L)$, on a un morphisme $\Phi_{L}: X \rightarrow \mathbb{P}(V)$ génériquement fini. Il existe donc un fermé $Z_{1} \neq X$ tel que $\Phi_{L \mid X-Z_{1}}$ soit à fibres finies. On applique alors la version de Vojta (cf. théorème 2 de [17]) du théorème de Cartan :

Il existe une réunion finie $H$ d'hyperplans de $\mathbb{P}(V) \simeq \mathbb{P}_{\mathbb{C}}^{q-1}$ telle que pour toute courbe entière d'image non contenue dans $Z_{1} \cup \Phi_{L}^{-1}(H)$, l'ensemble des réels $r \geq 0$ vérifiant ( $1^{\prime}$ ) est de mesure de Lebesgue finie.

THÉORÈme 3.5. - Soient $D_{1} ; \ldots ; D_{r}$ des diviseurs effectifs non nuls sur $X$ qui se coupent proprement deux à deux. Posons $Y=X-D_{1} \cup \cdots \cup D_{r}$. Soit $m \geq 1$ un entier. On suppose que le diviseur $L=m \sum_{i=1}^{r} D_{i}$ est libre et gros sur $X$ et que $\nu\left(L ; D_{1} ; \ldots ; D_{r}\right)>m$. Alors $Y$ est Brody quasi-hyperbolique.

Démonstration. - On procède en deux étapes : dans la première, on construit un fermé $Z \neq X$ candidat à contenir toutes les courbes entières; dans la seconde, on prouve que $Y$ est Brody quasi-hyperbolique.

Étape 1. - On reprend la démonstration du théorème 3.3, jusqu'à la construction des bases $\mathcal{B}_{I ; \underline{a}}$. On munit chaque faisceau $\mathcal{O}_{X}\left(D_{i}\right)$ d'une métrique \|\| . Appliquons le théorème de Cartan et Vojta (proposition 3.4) avec $\left\{s_{1} ; \ldots ; s_{N}\right\}=\mathcal{B}_{0} \cup \bigcup_{I ; \underline{a}} \mathcal{B}_{I ; \underline{a}}$ :

Il existe un fermé $Z \neq X$ tel que pour toute courbe entière $f$ sur $X$ d'image non contenue dans $Z(\mathbb{C})$, l'ensemble des réels $r \geq 0$ vérifiant l'inégalité $\left(1^{\prime}\right)$ est de mesure de Lebesgue finie.

Étape 2. - Soit $f$ une courbe entière sur $Y$. Raisonnons par l'absurde en supposant $f(\mathbb{C}) \not \subset$ $Z(\mathbb{C})$.

Par compacité de $X(\mathbb{C})$, il existe un réel $M>0$ tel que pour tout $y \in X(\mathbb{C})$, l'ensemble d'indices $I_{y}=\left\{i \in\{1 ; \ldots ; r\} \mid-\ln \left\|1_{D_{i}}(y)\right\| \geq M\right\}$ appartienne à $\mathcal{P} \cup\{\varnothing\}$ (il suffit d'extraire du recouvrement ouvert $\left(\left\{y \in X(\mathbb{C}) \mid \exists I \in \mathcal{P} \cup\{\varnothing\} \forall i \notin I-\ln \left\|1_{D_{i}}(y)\right\|<M\right\}\right)_{M>0}$ un recouvrement fini).

FaIt. - Soit $y \in Y(\mathbb{C})$. Il existe une base $\left(s_{1 y} ; \ldots ; s_{q y}\right)$ de $\Gamma(X ; L)$ contenue dans $\left\{s_{1} ; \ldots ; s_{N}\right\}$ telle que l'on ait la minoration suivante:

$$
-\sum_{k=1}^{q} \ln \left\|s_{k y}(y)\right\| \geq-(q+3 q \varepsilon) \ln \left\|1_{L}(y)\right\|-O(1),
$$

où le $O(1)$ est indépendant de $y$.

Prouvons ce fait. Si $I_{y}$ est vide, on prend $\left\{s_{1 y} ; \ldots ; s_{q y}\right\}=\mathcal{B}_{0}$ et on obtient la minoration $\left(2^{\prime}\right)$ en remarquant que $-\ln \left\|1_{L}(y)\right\|<M r$.

On suppose maintenant $I_{y}$ non vide. On a donc $I_{y} \in \mathcal{P}$. Pour tout $i \in I_{y}$, on pose $t_{y i}=$ $\frac{\ln \left\|1_{D_{i}}(y)\right\|}{\sum_{j \in I_{y}} \ln \left\|1_{D_{j}}(y)\right\|}$. Remarquons que l'on a $\sum_{i \in I_{y}} t_{y i}=1$. Choisissons un $\underline{a}_{y}=\left(a_{y i}\right)_{i} \in \Delta_{I_{y}}$ tel que $\left|b t_{y i}-a_{y i}\right| \leq 1$ pour tout $i \in I_{y}$. On prend alors $\left\{s_{1 y} ; \ldots ; s_{q y}\right\}=\mathcal{B}_{I_{y} ; \underline{a}_{y}}$. Vérifions que ce choix convient. 
Soit $s \in \Gamma(X ; L)-\{0\}$. Puisque les diviseurs $D_{i}$ se coupent proprement deux à deux, le diviseur $\operatorname{div}(s)-\sum_{i \in I_{y}} \mu_{i}(s) D_{i}$ est effectif. Ceci implique

$$
-\ln \|s(y)\| \geq-\sum_{i \in I_{y}} \mu_{i}(s) \ln \left\|1_{D_{i}}(y)\right\|-O(1) .
$$

En remarquant que $t_{y i} \geq \frac{a_{y i}}{b}-\frac{m \varepsilon}{c r}$ et que $\mu_{i}(s) \leq c$ pour tout $i \in I_{y}$, on a, par définition des $t_{y i}$ :

$$
-\mu_{i}(s) \ln \left\|1_{D_{i}}(y)\right\| \geq-\left(\frac{a_{y i}}{b} \mu_{i}(s)-\frac{m \varepsilon}{r}\right) \sum_{j \in I_{y}} \ln \left\|1_{D_{j}}(y)\right\|
$$

pour tout $i \in I_{y}$.

On en déduit l'inégalité

$$
-\ln \|s(y)\| \geq-\left(\frac{1}{b} \sum_{i \in I_{y}} a_{y i} \mu_{i}(s)-m \varepsilon\right) \sum_{j \in I_{y}} \ln \left\|1_{D_{j}}(y)\right\|-O(1) .
$$

On écrit cette inégalité pour $s=s_{k y}$, puis on somme sur $k$. En observant que pour $i \in I_{y}$, on a

$$
\sum_{k=1}^{q} \sum_{i \in I_{y}} a_{y i} \mu_{i}\left(s_{k y}\right) \geq \nu\left(L ; D_{1} ; \ldots ; D_{r}\right) q b=(1+4 \varepsilon) q b m
$$

comme dans la démonstration du théorème 3.3, on trouve alors

$$
-\sum_{k=1}^{q} \ln \left\|s_{k y}(y)\right\| \geq-(q+3 q \varepsilon) m \sum_{j \in I_{y}} \ln \left\|1_{D_{j}}(y)\right\|-O(1) .
$$

Le fait énoncé $\left(2^{\prime}\right)$ s'en déduit en remarquant que $-\ln \left\|1_{D_{j}}(y)\right\|<M$ pour tout $j \notin I_{y}$.

Maintenant $f$ est une courbe entière sur $Y$, donc pour tout $r \geq 0$, on a

$$
\mathrm{T}_{\hat{L} ; f}(r)=-\int_{0}^{2 \pi} \ln \left\|1_{L}\left(f\left(r e^{i \theta}\right)\right)\right\| \frac{\mathrm{d} \theta}{2 \pi}+O(1) .
$$

En utilisant la minoration $\left(2^{\prime}\right)$, on obtient (pour tout $r \geq 0$ )

$$
\int_{0}^{2 \pi} \max _{J \in \mathcal{L}} \sum_{k \in J} \ln \left\|s_{k}\left(f\left(r e^{i \theta}\right)\right)\right\|^{-1} \frac{\mathrm{d} \theta}{2 \pi} \geq(q+3 q \varepsilon) \mathrm{T}_{\hat{L} ; f}(r)-O(1) .
$$

D'où une contradiction avec $\left(1^{\prime}\right)$ (puisque $\mathrm{T}_{\hat{L} ; f}(r)$ tend vers $+\infty$ lorsque $r$ tend vers $+\infty$ ).

\section{Démonstration du théorème 2.1}

Soient $K$ un corps de caractéristique nulle et $X$ une variété projective sur $K$ de dimension $d \geq 2$. Pour tous diviseurs $L_{1} ; \ldots ; L_{d}$ sur $X$, on désigne par $\left\langle L_{1} \cdots L_{d}\right\rangle$ leur nombre d'intersection. Lorsque $L$ est un diviseur sur $X$ tel que $q=h^{0}(X ; L) \geq 1$ et $E$ un diviseur effectif non nul sur $X$, on pose $\alpha(L ; E)=\frac{1}{q} \sum_{k \geq 1} h^{0}(X ; L-k E)$. 
Proposition 4.1. - Soit $L$ un diviseur sur $X$ tel que $q=h^{0}(X ; L) \geq 1$. Soient $D_{1} ; \ldots ; D_{r}$ des diviseurs effectifs non nuls sur $X$ qui se coupent proprement deux à deux; supposons que toute intersection de $\delta+1$ quelconques d'entre eux est vide (avec $2 \leq \delta \leq r$ ). On a alors

$$
\nu\left(L ; D_{1} ; \ldots ; D_{r}\right) \geq \frac{2}{\delta} \inf _{i} \alpha\left(L ; D_{i}\right)
$$

Démonstration. - On utilise les notations de la section 2.1. Lorsque $x$ est un réel, on désigne par $\lceil x\rceil$ le plus petit entier $\geq x$. Soient $I \in \mathcal{P}$ et $\underline{a} \in \mathbb{N}^{I}-\{0\}$. Quitte à réduire $I$, on peut supposer que $a_{i} \geq 1$ pour tout $i \in I$. Observons que $\# I \leq \delta$.

Si $I$ est un singleton $\{i\}$, alors $V_{I ; \underline{a} ; k}=\Gamma\left(X ; L-\left\lceil\frac{k}{a_{i}}\right\rceil D_{i}\right)$, donc on a bien

$$
\sum_{k \geq 1} V_{I ; \underline{a} ; k}=a_{i} \sum_{k \geq 1} h^{0}\left(X ; L-k D_{i}\right) \geq a_{i} q \frac{2}{\delta} \inf _{j} \alpha\left(L ; D_{j}\right) .
$$

On suppose maintenant $\# I \geq 2$. On choisit deux indices $j<l$ dans $I$ tels que $a_{l} \geq a_{j} \geq a_{i}$ pour tout $i \in I-\{j ; l\}$. Pour $\left(b_{1} ; b_{2}\right) \in \mathbb{N}^{2}$, on pose $W\left(b_{1} ; b_{2}\right)=\Gamma\left(X ; L-b_{1} D_{j}-b_{2} D_{l}\right)$.

Soit $k$ un entier $\geq 1$. L'espace vectoriel $V_{I ; a ; k}$ contient alors le sous-espace

$$
V_{k}^{\prime}=W\left(0 ;\left\lceil\frac{k}{a_{l}}\right\rceil\right)+\sum_{b=0}^{\left\lceil k / a_{l}\right\rceil-1} W\left(\left\lceil\frac{k-a_{l} b}{a_{j}}\right\rceil ; b\right) .
$$

Puisque les diviseurs $D_{j}$ et $D_{l}$ se coupent proprement, on a l'égalité suivante pour tout $b^{\prime} \in\left\{0 ; \ldots ;\left\lceil k / a_{l}\right\rceil-1\right\}:$

$$
W\left(\left\lceil\frac{k-a_{l} b^{\prime}}{a_{j}}\right\rceil ; b^{\prime}\right) \bigcap\left[W\left(0 ;\left\lceil\frac{k}{a_{l}}\right\rceil\right)+\sum_{b=b^{\prime}+1}^{\left\lceil k / a_{l}\right\rceil-1} W\left(\left\lceil\frac{k-a_{l} b}{a_{j}}\right\rceil ; b\right)\right]=W\left(\left\lceil\frac{k-a_{l} b^{\prime}}{a_{j}}\right\rceil ; b^{\prime}+1\right) .
$$

En utilisant $\left\lceil k / a_{l}\right\rceil$ fois la formule $\operatorname{dim}\left(W_{1}+W_{2}\right)=\operatorname{dim} W_{1}+\operatorname{dim} W_{2}-\operatorname{dim} W_{1} \cap W_{2}$, on obtient que la dimension de $V_{k}^{\prime}$ vaut

$$
\operatorname{dim} W\left(0 ;\left\lceil\frac{k}{a_{l}}\right\rceil\right)+\sum_{b=0}^{\left\lceil k / a_{l}\right\rceil-1}\left[\operatorname{dim} W\left(\left\lceil\frac{k-a_{l} b}{a_{j}}\right\rceil ; b\right)-\operatorname{dim} W\left(\left\lceil\frac{k-a_{l} b}{a_{j}}\right\rceil ; b+1\right)\right] .
$$

Maintenant, on somme sur $k$ l'égalité précédente. Après simplifications, on trouve

$$
\sum_{k \geq 1} \operatorname{dim} V_{k}^{\prime}=a_{l} \sum_{k \geq 1} h^{0}\left(X ; L-k D_{l}\right)+a_{j} \sum_{k \geq 1} h^{0}\left(X ; L-k D_{j}\right) .
$$

On en conclut la minoration

$$
\sum_{k \geq 1} \operatorname{dim} V_{I ; \underline{a} ; k} \geq \sum_{k \geq 1} \operatorname{dim} V_{k}^{\prime} \geq\left(a_{j}+a_{l}\right) q \inf _{i} \alpha\left(L ; D_{i}\right) \geq\left(\sum_{i \in I} a_{i}\right) q \frac{2}{\delta} \inf _{j} \alpha\left(L ; D_{j}\right) .
$$

D’où le résultat.

On aura besoin dans la suite d'une variante des « inégalités de Morse holomorphes » (cf. [4] §12 et [1]) : 
Lemme 4.2. - Soient $E$ un diviseur libre et gros sur $X$ et $L$ un diviseur sur $X$ tel que $L-E$ soit nef. Soit $\beta$ un réel $>0$. Pour tout couple d'entiers $(n ; k)$ vérifiant $1 \leq k \leq \beta n$, on a alors la minoration

$h^{0}(X ; n L-k E) \geq \frac{\left\langle L^{d}\right\rangle}{d !} n^{d}-\frac{\left\langle L^{d-1} E\right\rangle}{(d-1) !} n^{d-1} k+\frac{d-1}{d !}\left\langle L^{d-2} E^{2}\right\rangle n^{d-2} \min \left(k^{2} ; n^{2}\right)-O\left(n^{d-1}\right)$, où le $O$ ne dépend pas de $(n ; k)$.

Démonstration. - Les $O$ apparaissant dans cette preuve dépendent de $(K ; X ; E ; L ; \beta)$ mais pas de $(n ; k)$. On a deux cas.

Cas $k \leq n$. - La formule de Hirzebruch-Riemann-Roch donne que $\chi(X ; n L-k E)$ est une fonction polynomiale en $(n ; k)$ dont on peut expliciter la composante homogène dominante :

Pour tout $(n ; k)$ tel que $1 \leq k \leq n$, on a $\chi(X ; n L-k E)=\frac{1}{d !}\left\langle(n L-k E)^{d}\right\rangle+O\left(n^{d-1}\right)$.

Par ailleurs, d'après le théorème 1.4 .40 de [10] p. 69 (ou plutôt d'après sa démonstration), on a $h^{i}(X ; n L-k E)=O\left(n^{d-i}\right)$ pour tout $i \geq 1$, puisque $L$ et $L-E$ sont nefs. On a en particulier $h^{0}(X ; n L-k E)=\frac{1}{d !}\left\langle(n L-k E)^{d}\right\rangle+O\left(n^{d-1}\right)$.

Or un calcul montre (par multilinéarité) la formule

$$
\left\langle(n L-k E)^{d}\right\rangle=\left\langle L^{d}\right\rangle n^{d}-d\left\langle L^{d-1} E\right\rangle n^{d-1} k+\sum_{i=2}^{d}(i-1)\left\langle L^{i-2}(n L-k E)^{d-i} E^{2}\right\rangle n^{i-2} k^{2}
$$

(en effet, on l'obtient en écrivant la relation

$$
\left\langle L^{d-1} E\right\rangle n^{d-1} k-\left\langle L^{j-1}(n L-k E)^{d-j} E\right\rangle n^{j-1} k=\sum_{i=j+1}^{d}\left\langle L^{i-2}(n L-k E)^{d-i} E^{2}\right\rangle n^{i-2} k^{2}
$$

et en la sommant sur $j$ ).

L'inégalité de l'énoncé s'en déduit facilement : les diviseurs $L, n L-k E$ et $E$ sont nefs, donc on a $\left\langle L^{i-2}(n L-k E)^{d-i} E^{2}\right\rangle \geq 0$ pour tout $i \in\{2 ; \ldots ; d-1\}$.

Cas $k>n$. - D'après le théorème de Bertini (cf. corollaire 6.11 de [8] p. 89), il existe $s \in$ $\Gamma(X ; E)-\{0\}$ tel que $Z=\operatorname{div}(s)$ soit géométriquement intègre sur $K$.

Soit $i$ un entier tel que $n \leq i \leq \beta n$. On a la suite exacte de $\mathcal{O}_{X}$-modules suivante :

$$
0 \rightarrow \mathcal{O}_{X}(n L-(i+1) E) \rightarrow \mathcal{O}_{X}(n L-i E) \rightarrow \mathcal{O}_{X}(n L-i E)_{\mid Z} \rightarrow 0 .
$$

On en déduit une suite exacte en cohomologie qui fournit l'inégalité

$$
h^{0}(X ; n L-(i+1) E) \geq h^{0}(X ; n L-i E)-h^{0}\left(Z ;(n L-i E)_{\mid Z}\right) .
$$

En utilisant la majoration

$$
h^{0}\left(Z ;(n L-i E)_{\mid Z}\right) \leq h^{0}\left(Z ; n L_{\mid Z}\right)=\frac{\left\langle L^{d-1} E\right\rangle}{(d-1) !} n^{d-1}+O\left(n^{d-2}\right)
$$

(obtenue par Hirzebruch-Riemann-Roch), on trouve

$$
\begin{aligned}
h^{0}(X ; n L-k E) & \geq h^{0}(X ; n L-n E)-\sum_{i=n}^{k-1} h^{0}\left(Z ;(n L-i E)_{\mid Z}\right) \\
& \geq \frac{\left\langle L^{d}\right\rangle}{d !} n^{d}-\frac{\left\langle L^{d-1} E\right\rangle}{(d-1) !} n^{d-1} k+\frac{d-1}{d !}\left\langle L^{d-2} E^{2}\right\rangle n^{d}-O\left(n^{d-1}\right)
\end{aligned}
$$

(la minoration de $h^{0}(X ; n L-n E)$ est donnée par le premier cas). D’où le résultat. 
Remarque. - La démonstration fournit en fait une minoration de $h^{0}(X ; n L-k E)-$ $h^{1}(X ; n L-k E)$.

On note ici $g: \mathbb{R}_{+} \rightarrow \mathbb{R}_{+}$l'application continue définie par $g(\beta)=\frac{\beta^{3}}{3}$ si $\beta \leq 1$ et $g(\beta)=$ $\beta-\frac{2}{3}$ si $\beta \geq 1$.

Corollaire 4.3. - Soient $E$ un diviseur effectif libre et gros sur $X$ et $L$ un diviseur sur $X$ tel que $L-E$ soit nef. On a alors

$$
\liminf _{n \rightarrow+\infty} \frac{1}{n} \alpha(n L ; E) \geq \frac{\left\langle L^{d}\right\rangle}{2 d\left\langle L^{d-1} E\right\rangle}+(d-1) \frac{\left\langle L^{d-2} E^{2}\right\rangle}{\left\langle L^{d}\right\rangle} g\left(\frac{\left\langle L^{d}\right\rangle}{d\left\langle L^{d-1} E\right\rangle}\right) .
$$

Démonstration. - On pose $\beta=\frac{\left\langle L^{d}\right\rangle}{d\left\langle L^{d-1} E\right\rangle}$ et $M=(d-1)\left\langle L^{d-2} E^{2}\right\rangle$. Grâce au lemme 4.2, on a les estimations suivantes :

$$
\begin{aligned}
\sum_{k \geq 1} h^{0}(X ; n L-k E) & \geq \sum_{k=1}^{\lfloor\beta n\rfloor}\left(\frac{\left\langle L^{d}\right\rangle}{d !} n^{d}-\frac{\left\langle L^{d-1} E\right\rangle}{(d-1) !} n^{d-1} k+\frac{M}{d !} n^{d-2} \min \left(k^{2} ; n^{2}\right)\right)-O\left(n^{d}\right) \\
& =\left(\frac{\left\langle L^{d}\right\rangle}{d !} \beta-\frac{\left\langle L^{d-1} E\right\rangle}{(d-1) !} \frac{\beta^{2}}{2}+\frac{M}{d !} g(\beta)\right) n^{d+1}-O\left(n^{d}\right) .
\end{aligned}
$$

D'où la minoration $\alpha(n L ; E) \geq\left(\frac{\beta}{2}+\frac{M}{\left\langle L^{d}\right\rangle} g(\beta)\right) n-O(1)$.

Montrons maintenant le résultat principal de cette section :

THÉORÈme 4.4. - Soient $D_{1} ; \ldots ; D_{r}$ des diviseurs effectifs presque amples sur $X$. Il existe alors des entiers $m_{1} ; \ldots ; m_{r}$ tels qu'en posant $L=\sum_{i=1}^{r} m_{i} D_{i}$, on ait

$$
m_{i} \geq 1 \text { et } \liminf _{n \rightarrow+\infty} \frac{1}{n} \alpha\left(n L ; m_{i} D_{i}\right)>\frac{r}{2 d} \text { pour tout } i \in\{1 ; \ldots ; r\} \text {. }
$$

Démonstration. - On pose ici $\Delta=\left\{\left(t_{1} ; \ldots ; t_{r}\right) \in \mathbb{R}_{+}^{r} \mid t_{1}+\cdots+t_{r}=1\right\}$. Pour tout $t=\left(t_{1} ; \ldots ; t_{r}\right) \in \Delta$, on désigne par $L_{t}$ le $\mathbb{R}$-diviseur $L_{t}=\sum_{j=1}^{r} t_{j} D_{j}$ et on pose $\phi(t)=$ $\left(\sum_{i=1}^{r} \frac{1}{\left\langle L_{t}^{d-1} D_{i}\right\rangle}\right)^{-1}$.

On note $f: \Delta \rightarrow \Delta$ l'application continue définie par $f(t)=\left(\frac{\phi(t)}{\left\langle L_{t}^{d-1} D_{1}\right\rangle} ; \ldots ; \frac{\phi(t)}{\left\langle L_{t}^{d-1} D_{r}\right\rangle}\right)$ pour tout $t \in \Delta$. D'après le théorème de Brouwer, $f$ admet un point fixe $x=\left(x_{1} ; \ldots ; x_{r}\right)$. On a alors $\phi(x)=\left\langle L_{x}^{d-1} D_{i}\right\rangle x_{i}$ pour tout $i \in\{1 ; \ldots ; r\}$, donc $\phi(x) r=\left\langle L_{x}^{d}\right\rangle$.

On en déduit l'inégalité

$$
\frac{\left\langle L_{x}^{d}\right\rangle}{2 d\left\langle L_{x}^{d-1} D_{i}\right\rangle x_{i}}+(d-1) \frac{\left\langle L_{x}^{d-2} D_{i}^{2}\right\rangle x_{i}^{2}}{\left\langle L_{x}^{d}\right\rangle} g\left(\frac{\left\langle L_{x}^{d}\right\rangle}{d\left\langle L_{x}^{d-1} D_{i}\right\rangle x_{i}}\right)>\frac{r}{2 d} \text { pour tout } i \in\{1 ; \ldots ; r\} .
$$

On approche $x$ par un $y \in \mathbb{Q}_{+}^{* r} \cap \Delta$ de la forme $y=\left(\frac{m_{1}}{m} ; \ldots ; \frac{m_{r}}{m}\right)$ de telle sorte que l'inégalité précédente soit encore valable avec $y$ au lieu de $x$, et on conclut en appliquant le corollaire 4.3.

On en déduit le théorème 2.1 en appliquant la proposition 4.1. 


\section{Géométrie bis}

\subsection{Préliminaires}

Soient $r$ et $m$ des entiers $\geq 1$. On pose $\Delta=\{0 ; \ldots ; m\}^{r}$. On munit $\Delta$ de l'ordre lexicographique. Notons $\underline{m}=(m ; \ldots ; m)$ le plus grand élément de $\Delta$. Pour tout $\underline{b}=\left(b_{1} ; \ldots ; b_{r}\right) \in \Delta$, on désigne par $J_{\underline{b}}$ l'ensemble des $i \in\{1 ; \ldots ; r\}$ tels que $b_{i}<m$.

Commençons par la variante suivante du lemme 2.2 de [2] :

Lemme 5.1. - Soit A un anneau local. Soit $\left(\varphi_{1} ; \ldots ; \varphi_{r}\right)$ une suite régulière de A. Pour tout $\underline{b} \in \Delta$, on a alors l'inclusion d'idéaux

$$
\left(\varphi_{1}^{b_{1}} \cdots \varphi_{r}^{b_{r}} A\right) \cap\left(\sum_{\underline{c}>\underline{b}} \varphi_{1}^{c_{1}} \cdots \varphi_{r}^{c_{r}} A\right) \subset \sum_{j \in J_{\underline{b}}} \varphi_{1}^{b_{1}} \cdots \varphi_{r}^{b_{r}} \varphi_{j} A .
$$

Démonstration. - On raisonne par récurrence sur $r$. Si $r=1$, alors l'inclusion est évidente. Supposons $r \geq 2$ et le résultat au cran $r-1$. Posons $\Delta^{\prime}=\{0 ; \ldots ; m\}^{r-1}$ et $\underline{b}^{\prime}=$ $\left(b_{2} ; \ldots ; b_{r}\right)$. Soit $x \in\left(\varphi_{1}^{b_{1}} \cdots \varphi_{r}^{b_{r}} A\right) \cap\left(\sum_{\underline{c}>\underline{b}} \varphi_{1}^{c_{1}} \cdots \varphi_{r}^{c_{r}} A\right)$. On a deux cas.

Cas $b_{1}=m$. - L'élément $x$ s'écrit $x=\varphi_{1}^{m} y=\sum_{\underline{c}^{\prime} \underline{b}^{\prime}} \varphi_{1}^{m} a_{\underline{c}^{\prime}}$ avec un $y \in \varphi_{2}^{b_{2}} \cdots \varphi_{r}^{b_{r}} A$ et des $a_{\underline{c}^{\prime}} \in \varphi_{2}^{c_{2}} \cdots \varphi_{r}^{c_{r}} A$. En simplifiant par $\varphi_{1}^{m}$, on obtient que $y$ appartient à $\sum_{\underline{c}^{\prime}>\underline{b}^{\prime}} \varphi_{2}^{c_{2}} \cdots \varphi_{r}^{c_{r}} A$. Or $\left(\varphi_{2} ; \ldots ; \varphi_{r}\right)$ est une suite régulière de $A$, donc $y$ est un élément de $\sum_{j \in J_{\underline{b}}} \varphi_{2}^{b_{2}} \cdots \varphi_{r}^{b_{r}} \varphi_{j} A$ par hypothèse de récurrence.

Cas $b_{1}<m$. - L'élément $x$ s'écrit $x=\varphi_{1}^{b_{1}} y=\varphi_{1}^{b_{1}+1} z+\sum_{\underline{c}^{\prime}>\underline{b}^{\prime}} \varphi_{1}^{b_{1}} a_{\underline{c}^{\prime}}$ avec un $y \in$ $\varphi_{2}^{b_{2}} \cdots \varphi_{r}^{b_{r}} A$, un $z \in A$ et des $a_{\underline{c}^{\prime}} \in \varphi_{2}^{c_{2}} \cdots \varphi_{r}^{c_{r}} A$. On écrit $y=\varphi_{2}^{b_{2}} \cdots \varphi_{r}^{b_{r}} w$ avec $w \in A$. On simplifie par $\varphi_{1}^{b_{1}}$ puis on réduit modulo $\varphi_{1}$; on trouve ainsi dans $A^{\prime}=A / \varphi_{1} A$ l'égalité $\bar{y}=\sum_{\underline{c}^{\prime}>\underline{b^{\prime}}} \overline{a_{\underline{c}^{\prime}}}$.

On en déduit que $\bar{y}$ appartient à $\left({\overline{\varphi_{2}}}^{b_{2}} \cdots \overline{\varphi_{r}}{ }^{b_{r}} A^{\prime}\right) \cap\left(\sum_{\underline{c}^{\prime}>\underline{b^{\prime}}}{\overline{\varphi_{2}}}^{c_{2}} \cdots \overline{\varphi_{r}} \bar{c}_{r} A^{\prime}\right)$. Or $\left(\overline{\varphi_{2}} ; \ldots ; \overline{\varphi_{r}}\right)$ est une suite régulière de $A^{\prime}$, donc $\bar{y}$ est un élément de $\sum_{j \in J_{b}-\{1\}} \overline{\varphi_{2}}{ }^{b_{2}} \cdots \overline{\varphi_{r}}{ }^{b_{r}} \overline{\varphi_{j}} A^{\prime}$ par hypothèse de récurrence. En simplifiant par $\overline{\varphi_{2}} b_{2} \cdots \overline{\varphi_{r}}{ }^{b_{r}}$, on obtient que $\bar{w}$ est dans $\sum_{j \in J_{\underline{b}}-\{1\}} \bar{\varphi}_{j} A^{\prime}$. On en conclut que $w$ appartient à $\sum_{j \in J_{\underline{b}}} \varphi_{j} A$.

D'où le résultat.

Soient $K$ un corps de caractéristique nulle et $X$ une variété projective sur $K$ de dimension $d \geq 1$.

Définition. - Un $\mathcal{O}_{X}$-module cohérent $\mathcal{C}$ sur $X$ est dit acyclique lorsque $h^{i}(X ; \mathcal{C})=0$ pour tout $i \geq 1$.

Soit $L$ un diviseur sur $X$ tel que $q=h^{0}(X ; L) \geq 1$. Soient $D_{1} ; \ldots ; D_{r}$ des diviseurs effectifs non nuls sur $X$ qui se coupent proprement.

Pour tout $\underline{b} \in \Delta$, on pose $\mathcal{L}_{\underline{b}}=\mathcal{O}_{X}\left(L-\sum_{i=1}^{r} b_{i} D_{i}\right)$. Pour $\underline{b} \in \Delta$, on définit le sousmodule $\mathcal{C}_{\underline{b}}$ de $\mathcal{L}_{\underline{b}}$ par

$$
\mathcal{C}_{\underline{b}}=\sum_{j \in J_{\underline{b}}} \mathcal{O}_{X}\left(L-D_{j}-\sum_{i=1}^{r} b_{i} D_{i}\right)
$$


Soit $\left(a_{1} ; \ldots ; a_{r}\right) \in \mathbb{N}^{r}$. Pour $k \in \mathbb{N}^{*}$, on note $V_{k}$ le sous-espace de $\Gamma(X ; L)$ défini par

$$
V_{k}=\sum_{\underline{b}} \Gamma\left(X ; \mathcal{L}_{\underline{b}}\right) \text { où la somme porte sur les } \underline{b} \in \mathbb{N}^{r} \text { tels que } \sum_{i=1}^{r} a_{i} b_{i} \geq k .
$$

Lemme 5.2. - Avec ces notations, on a la minoration suivante:

$$
\sum_{k \geq 1} \operatorname{dim} V_{k} \geq \sum_{i=1}^{r} a_{i} \sum_{\underline{b} \in \Delta}\left[h^{0}\left(X ; \mathcal{L}_{\underline{b}}\right)-h^{0}\left(X ; \mathcal{C}_{\underline{b}}\right)\right] b_{i} .
$$

Démonstration. - Soit $k$ un entier tel que $1 \leq k \leq \sum_{i=1}^{r} a_{i} m$. Notons $\mathcal{D}_{k}$ l'ensemble des $\underline{b} \in \Delta$ tels que $\sum_{i=1}^{r} a_{i} b_{i} \geq k$. L'espace vectoriel $V_{k}$ contient alors le sous-espace $V_{k}^{\prime}=$ $\sum_{\underline{b} \in \mathcal{D}_{k}} \Gamma\left(X ; \mathcal{L}_{\underline{b}}\right)$.

Soit $\underline{b} \in \mathcal{D}_{k}-\{\underline{m}\}$. Le lemme 5.1 fournit l'inclusion de $\mathcal{O}_{X}$-modules $\mathcal{L}_{\underline{b}} \cap \sum_{\underline{c}>\underline{b}} \mathcal{L}_{\underline{c}} \subset$ $\mathcal{C}_{\underline{b}}$, puisque les diviseurs $D_{1} ; \ldots ; D_{r}$ se coupent proprement. On a en particulier l'inclusion d'espaces vectoriels

$$
\Gamma\left(X ; \mathcal{L}_{\underline{b}}\right) \cap \sum_{\underline{c}>\underline{b}} \Gamma\left(X ; \mathcal{L}_{\underline{c}}\right) \subset \Gamma\left(X ; \mathcal{C}_{\underline{b}}\right) .
$$

En utilisant \#讘 -1 fois la formule $\operatorname{dim}\left(W_{1}+W_{2}\right)=\operatorname{dim} W_{1}+\operatorname{dim} W_{2}-\operatorname{dim} W_{1} \cap W_{2}$, on trouve l'inégalité

$$
\operatorname{dim} V_{k}^{\prime} \geq h^{0}\left(X ; \mathcal{L}_{\underline{m}}\right)+\sum_{\underline{b} \in \mathcal{D}_{k}-\{\underline{m}\}}\left[h^{0}\left(X ; \mathcal{L}_{\underline{b}}\right)-h^{0}\left(X ; \mathcal{C}_{\underline{b}}\right)\right] .
$$

On obtient le résultat en sommant sur $k$ cette inégalité.

Proposition 5.3. - On suppose de plus que $\mathcal{L}_{\underline{b}}$ est acyclique pour tout $\underline{b} \in \Delta$. On a alors

$$
\sum_{k \geq 1} \operatorname{dim} V_{k} \geq \sum_{i=1}^{r} a_{i} \sum_{k=1}^{m} h^{0}\left(X ; L-k D_{i}\right)
$$

On a en particulier $\nu\left(L ; D_{1} ; \ldots ; D_{r}\right) \geq \frac{1}{q} \inf _{i} \sum_{k=1}^{m} h^{0}\left(X ; L-k D_{i}\right)$.

Démonstration. - Soit $\underline{b} \in \Delta-\{\underline{m}\}$. Pour toute partie $I$ de $J_{\underline{b}}$, posons ici

$$
\mathcal{L}_{\underline{b} ; I}=\mathcal{O}_{X}\left(L-\sum_{j \in J_{\underline{b}}} D_{j}-\sum_{i=1}^{r} b_{i} D_{i}\right) \text {. }
$$

On pose aussi $p=\# J_{\underline{b}}$ et $\mathcal{E}_{\underline{b}}=\bigoplus_{j \in J_{\underline{b}}} \mathcal{L}_{\underline{b} ;\{j\}}$.

Les diviseurs $\left(D_{j}\right)_{j \in J_{\underline{b}}}$ se coupent proprement, donc on a la suite exacte de Koszul suivante (cf. [7] p. 431) :

$$
0 \rightarrow \Lambda^{p} \mathcal{E}_{\underline{b}} \rightarrow \cdots \rightarrow \Lambda^{1} \mathcal{E}_{\underline{b}} \rightarrow \mathcal{C}_{\underline{b}} \rightarrow 0 .
$$

On remarque que $\Lambda^{j} \mathcal{E}_{\underline{b}}=\bigoplus_{\# I=j} \mathcal{L}_{\underline{b} ; I}$ (qui est en particulier acyclique) pour tout $j \in$ $\{1 ; \ldots ; p\}$. La suite de Koszul précédente induit donc par acyclicité une suite exacte en image directe :

$$
0 \rightarrow \Gamma\left(X ; \Lambda^{p} \mathcal{E}_{\underline{b}}\right) \rightarrow \cdots \rightarrow \Gamma\left(X ; \Lambda^{1} \mathcal{E}_{\underline{b}}\right) \rightarrow \Gamma\left(X ; \mathcal{C}_{\underline{b}}\right) \rightarrow 0 .
$$

On en déduit la relation

$$
h^{0}\left(X ; \mathcal{L}_{\underline{b}}\right)-h^{0}\left(X ; \mathcal{C}_{\underline{b}}\right)=\sum_{I \subset J_{\underline{b}}}(-1)^{\# I} h^{0}\left(X ; \mathcal{L}_{\underline{b} ; I}\right) .
$$


Maintenant, on fixe $i \in\{1 ; \ldots ; r\}$ et $c \in\{0 ; \ldots ; m\}$, et on somme sur l'ensemble $\Delta_{c}^{\prime}$ des $\underline{b} \in \Delta$ tels que $b_{i}=c$. Un réarrangement des termes permet de simplifier et montre que :

$$
\sum_{\underline{b} \in \Delta_{c}^{\prime}} \sum_{I \subset J_{\underline{b}}}(-1)^{\# I} h^{0}\left(X ; \mathcal{L}_{\underline{b} ; I}\right)= \begin{cases}h^{0}\left(X ; L-c D_{i}\right)-h^{0}\left(X ; L-(c+1) D_{i}\right) & \text { si } c<m ; \\ h^{0}\left(X ; L-m D_{i}\right) & \text { si } c=m .\end{cases}
$$

(En effet, si $p^{\prime}=\#\left\{j \neq i \mid b_{j} \geq 1\right\} \geq 1$, alors le terme $h^{0}\left(X ; \mathcal{L}_{\underline{b}}\right)$ apparait $2^{p^{\prime}-1}$ fois avec le signe plus et $2^{p^{\prime}-1}$ fois avec le signe moins; de même avec le terme $h^{0}\left(X ; \mathcal{L}_{\underline{b} ;\{i\}}\right)$ dans le cas $c<m)$.

On en déduit l'égalité

$$
\begin{aligned}
\sum_{\underline{b} \in \Delta}\left[h^{0}\left(\mathcal{L}_{\underline{b}}\right)-h^{0}\left(\mathcal{C}_{\underline{b}}\right)\right] b_{i} & =h^{0}\left(L-m D_{i}\right) m+\sum_{c=0}^{m-1}\left[h^{0}\left(L-c D_{i}\right)-h^{0}\left(L-(c+1) D_{i}\right)\right] c \\
& =\sum_{k=1}^{m} h^{0}\left(X ; L-k D_{i}\right) .
\end{aligned}
$$

On conclut en appliquant le lemme 5.2.

\subsection{Démonstration du théorème 2.2}

Soient $K$ un corps de caractéristique nulle et $X$ une variété projective sur $K$ de dimension $d \geq 1$. Soient $D_{1} ; \ldots ; D_{r}$ des diviseurs effectifs non nuls sur $X$ qui se coupent proprement (avec $r \geq 1$ ). Notons $\mathcal{P}$ l'ensemble des parties $I$ non vides de $\{1 ; \ldots ; r\}$ telles que $\bigcap_{i \in I} D_{i}$ soit non vide.

ThÉORÈme 5.4. - Soit $L$ un diviseur ample sur $X$. On suppose que $D_{i}$ est nef pour tout $i \in\{1 ; \ldots ; r\}$. Soit $\theta>1$ un réel tel que le $\mathbb{R}$-diviseur $L-\theta \sum_{i \in I} D_{i}$ soit nef pour tout $I \in \mathcal{P}$. On a alors l'inégalité

$$
\liminf _{n \rightarrow+\infty} \frac{1}{n} \nu\left(n L ; D_{1} ; \ldots ; D_{r}\right) \geq \frac{\theta}{(d+1)\left\langle L^{d}\right\rangle} \inf _{i} \sum_{j=0}^{d}\left\langle L^{d-j}\left(L-\theta D_{i}\right)^{j}\right\rangle .
$$

Démonstration. - D'après le théorème d'annulation de Fujita (cf. théorème 1.4.35 de [10] p. 66), il existe $n_{0} \geq 1$ tel que $n_{0} L+N$ soit acyclique pour tout $N \in \operatorname{Pic}(X)$ nef.

On pose $n^{\prime}=\left\lfloor\left(n-n_{0}\right) \theta\right\rfloor$ pour tout $n>n_{0}$. En appliquant la proposition 5.3 (avec $m=n^{\prime}$ ), on obtient (pour tout $n>n_{0}$ )

$$
\nu\left(n L ; D_{1} ; \ldots ; D_{r}\right) \geq \frac{1}{h^{0}(X ; n L)} \inf _{i} \sum_{k=1}^{n^{\prime}} h^{0}\left(X ; n L-k D_{i}\right) .
$$


Soit $i \in\{1 ; \ldots ; r\}$. Grâce à la formule de Hirzebruch-Riemann-Roch, on a les estimations suivantes :

$$
\begin{aligned}
\sum_{k=1}^{n^{\prime}} h^{0}\left(X ; n L-k D_{i}\right) & =\frac{1}{d !} \sum_{k=1}^{n^{\prime}}\left[\left\langle\left(n L-k D_{i}\right)^{d}\right\rangle+O\left(n^{d-1}\right)\right] \\
& =\frac{1}{d !} \sum_{j=0}^{d} \sum_{k=1}^{n^{\prime}} \mathrm{C}_{d}^{j}\left\langle L^{d-j} D_{i}^{j}\right\rangle n^{d-j}(-k)^{j}+O\left(n^{d}\right) \\
& =\frac{1}{d !} \sum_{j=0}^{d} \mathrm{C}_{d}^{j}\left\langle L^{d-j} D_{i}^{j}\right\rangle \frac{(-1)^{j}}{j+1} \theta^{j+1} n^{d+1}+O\left(n^{d}\right) .
\end{aligned}
$$

Or un calcul montre la formule

$$
\sum_{j=0}^{d} \mathrm{C}_{d}^{j}\left\langle L^{d-j} D_{i}^{j}\right\rangle \frac{(-1)^{j}}{j+1} \theta^{j+1}=\frac{\theta}{d+1} \sum_{j=0}^{d}\left\langle L^{d-j}\left(L-\theta D_{i}\right)^{j}\right\rangle .
$$

D'où l'inégalité de l'énoncé.

Corollaire 5.5. - Soit $L$ un diviseur ample sur $X$. On suppose que $D_{i}$ est nef pour tout $i \in\{1 ; \ldots ; r\}$. Soit $\theta>1$ un réel tel que le $\mathbb{R}$-diviseur $L-d \theta D_{i}$ soit nef pour tout $i \in\{1 ; \ldots ; r\}$. On a alors

$$
\liminf _{n \rightarrow+\infty} \frac{1}{n} \nu\left(n L ; D_{1} ; \ldots ; D_{r}\right) \geq \lambda_{d} \theta .
$$

Démonstration. - Pour tout $I \in \mathcal{P}$, le $\mathbb{R}$-diviseur $L-\theta \sum_{i \in I} D_{i}$ est nef puisque $\# I \leq d$. Soit $i \in\{1 ; \ldots ; r\}$. Les $\mathbb{R}$-diviseurs $L-\theta D_{i}-\left(1-\frac{1}{d}\right) L$ et $L$ sont nefs, donc on a

$$
\left\langle L^{d-j}\left(L-\theta D_{i}\right)^{j}\right\rangle \geq\left(1-\frac{1}{d}\right)^{j}\left\langle L^{d}\right\rangle \text { pour tout } j \in\{1 ; \ldots ; d\} .
$$

En appliquant le théorème 5.4, on trouve ainsi

$$
\liminf _{n \rightarrow+\infty} \frac{1}{n} \nu\left(n L ; D_{1} ; \ldots ; D_{r}\right) \geq \frac{\theta}{d+1} \sum_{j=0}^{d}\left(1-\frac{1}{d}\right)^{j}=\lambda_{d} \theta .
$$

D'où le résultat.

\section{RÉFÉRENCES}

[1] F. Angelini, An algebraic version of Demailly's asymptotic Morse inequalities, Proc. Amer. Math. Soc. 124 (1996), 3265-3269.

[2] P. Corvaja, U. Zannier, On a general Thue's equation, Amer. J. Math. 126 (2004), 1033-1055.

[3] P. Corvaja, U. Zannier, On integral points on surfaces, Ann. of Math. 160 (2004), 705-726.

[4] J.-P. Demailly, $L^{2}$ vanishing theorems for positive line bundles and adjunction theory, in Transcendental methods in algebraic geometry (Cetraro, 1994), Lecture Notes in Math. 1646, Springer, 1996, 1-97. 
[5] G. Faltings, Endlichkeitssätze für abelsche Varietäten über Zahlkörpern, Invent. Math. 73 (1983), 349-366.

[6] G. Faltings, Diophantine approximation on abelian varieties, Ann. of Math. 133 (1991), 549-576.

[7] W. Fulton, Intersection theory, $2^{\mathrm{e}}$ éd., Ergebnisse der Mathematik und ihrer Grenzgebiete 2, Springer, 1998.

[8] J.-P. Jounnolou, Théorèmes de Bertini et applications, Progress in Mathematics 42 , Birkhäuser, 1983.

[9] S. LANG, Number theory. III, Encyclopaedia of Mathematical Sciences 60, Springer, 1991.

[10] R. LAZArsfeld, Positivity in algebraic geometry. I, Ergebnisse der Mathematik und ihrer Grenzgebiete. 3. Folge. A Series of Modern Surveys in Mathematics 48, Springer, 2004.

[11] A. Levin, Generalizations of Siegel's and Picard's theorems, à paraître dans Annals of Math. arXiv:math.NT/0503699.

[12] H. P. Schlickewei, The p-adic Thue-Siegel-Roth-Schmidt theorem, Arch. Math. (Basel) 29 (1977), 267-270.

[13] W. M. Schmidt, Diophantine approximation, Lecture Notes in Math. 785, Springer, 1980.

[14] P. Vojta, Diophantine approximations and value distribution theory, Lecture Notes in Math. 1239, Springer, 1987.

[15] P. Vojta, A refinement of Schmidt's subspace theorem, Amer. J. Math. 111 (1989), 489518.

[16] P. Vojta, Integral points on subvarieties of semiabelian varieties. I, Invent. Math. 126 (1996), 133-181.

[17] P. Vojta, On Cartan's theorem and Cartan's conjecture, Amer. J. Math. 119 (1997), 117.

[18] S. Zhang, Small points and adelic metrics, J. Algebraic Geom. 4 (1995), 281-300.

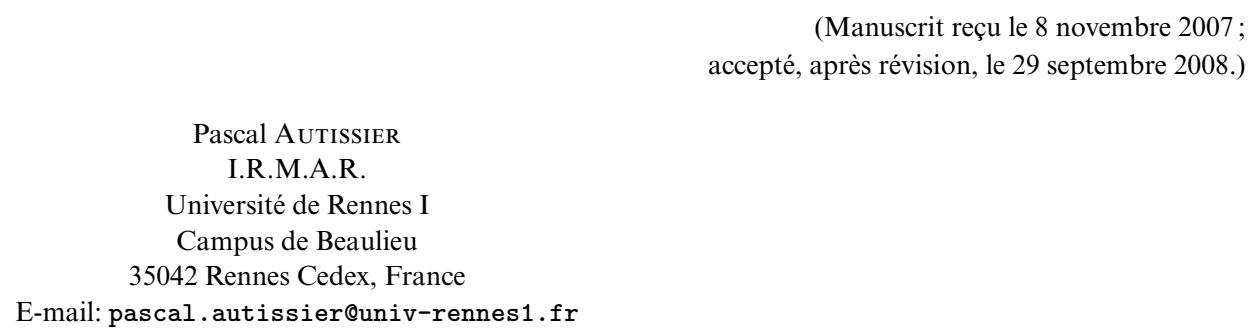

